\title{
Numerical Approximation of Dirichlet-to-Neumann Mapping and its Application to Voice Generation Problem
}

Takashi Kako and Kentarou Touda

The University of Electro-Communications, Department of Computer Science

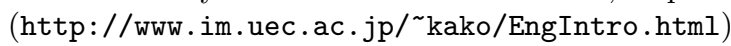

Summary. In this paper, we treat the numerical method for the Helmholtz equation in unbounded region with simple cylindrical or spherical shape outside some bounded region and apply the method to voice generation problem. The numerical method for the Helmholtz equation in unbounded region is based on the domain decomposition technique to divide the region into a bounded region and the rest unbounded one. We then treat the approximation of the artificial boundary condition given through the DtN mapping on the artificial boundary. We apply the finite element approximation to discretize the problem. In applying the method to the voice generation problem, it is essential to compute the frequency response function or the formant curve. We give variational formulas for the resolvent poles with respect to the variation of vocal tract boundary which determine the peaks of frequency response function known as formants, and we propose the use of variational formulas to design the location of formants.

\section{Numerical Method for Exterior Helmholtz Problem}

\subsection{Formulation of Exterior Helmholtz Problem}

We consider the following 2-dimensional exterior Helmholtz equation with unknown function $u$ as the mathematical model for the time stationary problem of outgoing or radiation sound wave propagation in unbounded region outside an obstacle:

$$
\begin{aligned}
-\Delta u-k^{2} u & =0 & & \text { in } \Omega=R^{2} \backslash \mathcal{O}, \\
\frac{\partial u}{\partial n} & =g & & \text { on } \partial \Omega, \\
\lim _{r \rightarrow \infty} \sqrt{r}\left(\frac{\partial u}{\partial r}-\mathbf{i} k u\right) & =0, & & \mathbf{i}=\sqrt{-1},
\end{aligned}
$$

where $\Omega$ is the interior of the complement of a bounded obstacle $\mathcal{O}$ in $R^{2}$ with smooth boundary $\partial \Omega$ on which the Neumann boundary condition (2) is 
imposed with an inhomogeneous data $g$. A real constant $k$ is called a wave number and the condition (3) is the Sommerfeld radiation condition at infinity which excludes any unphysical incoming wave. In the case with tubular cylindrical outside region, a similar formulation is possible with necessary modification of boundary condition and radiation condition (see Section 2.2).

Related to this problem in unbounded region, we introduce a circular artificial boundary $\Gamma_{R}$ with radius $R$ and decompose the original domain into two sub-domains. We then consider the boundary value problem in the part of bounded sub-domain given as

$$
\begin{aligned}
-\Delta u-k^{2} u & =0 & & \text { in } \Omega_{R} \equiv \Omega \cap B_{R}, \\
\frac{\partial u}{\partial n} & =g & & \text { on } \partial \Omega, \\
\frac{\partial u}{\partial r} & =M u & & \text { on } \Gamma_{R},
\end{aligned}
$$

where $B_{R} \supset \supset \mathcal{O}$ is a circular domain with radius $R$ bounded by an artificial circular boundary $\Gamma_{R}: B_{R}=\left\{(x, y) \mid r \equiv \sqrt{x^{2}+y^{2}}<R\right\}$, and $M$ is a differential or pseudo-differential operator which we construct as a function of the operator $\partial^{2} / \partial \theta^{2}$ of angular variable $\theta$ in order to make the problem exactly or approximately equivalent to the original problem.

The exact solution $u(r, \theta)$ for (1)-(3) in $B_{R}^{c}$ is given by the following formula with the Hankel function of the first kind of order $n$ which we will denote in this paper by $H^{(1)}(\cdot ; n)\left(=H_{n}^{(1)}(\cdot)\right)$ :

$$
u(r, \theta)=\frac{1}{2 \pi} \sum_{n=-\infty}^{\infty} \frac{H^{(1)}(k r ; n)}{H^{(1)}(k R ; n)} \int_{0}^{2 \pi} u(R, \phi) e^{\mathrm{i} n(\theta-\phi)} d \phi .
$$

Using this expression, we introduce the exact Dirichlet-to-Neumann (DtN) mapping as:

$$
M_{\text {exact }} u(\theta) \equiv \frac{k}{2 \pi} \sum_{n=-\infty}^{\infty} \frac{H^{(1)^{\prime}}(k R ; n)}{H^{(1)}(k R ; n)} \int_{0}^{2 \pi} u(R, \phi) e^{\mathbf{i} n(\theta-\phi)} d \phi,
$$

which relates the Dirichlet data of the solution $u$ on the artificial boundary $\Gamma_{R}$ to the Neumann data on the same boundary. If we put $M=M_{\text {exact }}$ in (6), the problem (4)-(6) is equivalent to the original problem (1)-(3). The DtN mapping can also be expressed as the function of the elliptic operator $D^{2}$ as:

$$
M_{\text {exact }}=M_{D t N}\left(D^{2}\right)=k \frac{H^{(1)^{\prime}}\left(k R ; \sqrt{D^{2}}\right)}{H^{(1)}\left(k R ; \sqrt{D^{2}}\right)}, \quad D \equiv-\mathbf{i} \partial / \partial \theta .
$$

\subsection{Radiation Boundary Conditions}

There have been many studies related to the analytical as well as numerical approximations of the DtN mapping. Among them, Engquist and Majda 
[1977], Engquist and Majda [1979] introduced a series of non-local approximate radiation boundary condition such as:

$$
\begin{aligned}
& M_{1}\left(D^{2}\right)=\frac{\mathbf{i}}{R} \sqrt{k^{2} R^{2}-D^{2}} \\
& M_{2}\left(D^{2}\right)=M_{1}\left(D^{2}\right)-\frac{1}{2 R} \frac{k^{2} R^{2}}{\left(k^{2} R^{2}-D^{2}\right)}
\end{aligned}
$$

and so forth. Some local approximate radiation boundary conditions are well used and they are given as

$$
\begin{aligned}
& M_{1,1}\left(D^{2}\right)=\mathbf{i} k \\
& M_{2,1}\left(D^{2}\right)=\mathbf{i} k-\frac{1}{2 R} .
\end{aligned}
$$

Those are derived directly from the Sommerfeld radiation condition. Feng [1983] introduced a series of local type operators such as

$$
\begin{aligned}
& F_{3}\left(D^{2}\right)=\mathbf{i} k-\frac{1}{2 R}+\frac{\mathbf{i}}{8 k R^{2}}-\frac{\mathbf{i}}{2 k R^{2}} D^{2} \\
& F_{4}\left(D^{2}\right)=\mathbf{i} k-\frac{1}{2 R}+\frac{\mathbf{i}}{8 k R^{2}}+\frac{1}{8 k^{2} R^{3}}-\left(\frac{\mathbf{i}}{2 k R^{2}}+\frac{1}{2 k^{2} R^{3}}\right) D^{2} .
\end{aligned}
$$

Bayliss and Turkel [1980] also introduced in a systematic way a hierarchy of local operators:

$$
L_{n}=\prod_{j=1}^{n}\left(\frac{\partial}{\partial r}-\mathbf{i} k+\frac{4 j-3}{2 r}\right), n \geq 1
$$

On the other hand, related to the finite element method, Kako and Kano [1999] proposed a non-local approximation by a bounded operator as a higher order correction:

$$
M_{L K}\left(D^{2}\right)=\mathbf{i} k-\frac{3}{2 R}+\frac{1}{R}\left[1+\frac{1}{2 \mathbf{i} k R}\left(\frac{1}{4}-D^{2}\right)\right]^{-1} .
$$

According to our numerical experiments, the exact DtN operator is the best one when it is combined with the appropriate discrete approximation. In the next subsection, we will briefly review the recent results of Nasir et al. [2003] based on a mixed method approximation.

\subsection{Finite Element Approximation}

To formulate the finite element method for the Helmholtz problem, we introduce a function spaces $V \equiv H^{1}\left(\Omega_{R}\right)$. Then the weak formulation of the problem is to find $u \in V$ such that 


$$
a(u, v)-\langle u, v\rangle_{M}=(g, v)_{\partial \Omega} \quad \forall v \in V
$$

with

$$
\begin{aligned}
a(u, v) & =\int_{\Omega_{R}}\left(\frac{\partial u}{\partial r} \frac{\partial \bar{v}}{\partial r}+\frac{1}{r^{2}} \frac{\partial u}{\partial \theta} \frac{\partial \bar{v}}{\partial \theta}-k^{2} u \bar{v}\right) r d r d \theta \\
\langle u, v\rangle_{M} & =\int_{0}^{2 \pi}(M u) \bar{v} R d \theta, \quad(f, g)_{\partial \Omega}=\int_{\partial \Omega} f \bar{g} d \sigma
\end{aligned}
$$

where $M$ is one of the operators appeared in the previous section. Let us introduce a finite dimensional subspace $V_{h}$ of $V$. Then the finite element approximation is to find $u_{h} \in V_{h}$ such that

$$
a\left(u_{h}, v_{h}\right)-\left\langle u_{h}, v_{h}\right\rangle_{M}=\left(g, v_{h}\right)_{\partial \Omega}, \quad \forall v_{h} \in V_{h} .
$$

In the following, we will introduce the fictitious domain method combined with a fast direct method. For this purpose, we firstly treat a special problem with annulus region.

\subsection{Fast Direct Method}

In case that $\Omega_{R}$ is an annulus region, we can make a separation of variables with respect to the radial and angular coordinates, and by dividing the intervals into $n_{r}$ subintervals in radial direction and into $n_{\theta}$ in angular direction, the finite element method gives a linear system:

$$
\mathbf{B U}=\mathbf{F}
$$

with a separable matrix $\mathbf{B}=\left(b_{I J}\right)$, a given vector $\mathbf{f}=\left(f_{I}\right)$ and a solution vector $\mathbf{u}=\left(u_{J}\right)$ where $b_{I J}=a\left(\Phi_{J}, \Phi_{I}\right)-\left\langle\Phi_{J}, \Phi_{I}\right\rangle_{M}$ and $f_{I}=\left(g, \Phi_{I}\right)_{\partial \Omega}$. The matrix $\mathbf{B}$ is given by a tensor product form:

$$
\mathbf{B}=\mathbf{R}_{2} \otimes \mathbf{T}_{1}+\mathbf{R}_{1} \otimes \mathbf{T}_{2}-k^{2} \mathbf{R}_{1} \otimes \mathbf{T}_{1}-\mathbf{e}_{n_{r}}^{n_{r}} \mathbf{e}_{n_{r}}^{n_{r} T} \otimes \mathbf{M}
$$

with a tri-diagonal matrices $\mathbf{R}_{i} \in C^{n_{r} \times n_{r}}(i=1,2)$ and circulant matrices $\mathbf{T}_{i}(i=1,2)$ and $\mathbf{M} \in C^{n_{\theta} \times n_{\theta}}$. The matrix $\mathbf{M}$ corresponds to the radiation boundary condition and $\mathbf{e}_{j}^{n}$ denotes the usual $j$ th canonical basis vector of $R^{n}$. Explicit forms for the matrices for $\mathbf{R}_{i}, \mathbf{T}_{i}$ and $\mathbf{M}$ can be found, for example, in Ernst [1996].

To solve the system (11), a fast direct solution method based on separation of variables can be used by diagonalizing the circulant matrices. This leads to

$$
\begin{gathered}
\left(\mathbf{I}_{n_{r}} \otimes \mathbf{Q}^{H}\right)\left(\mathbf{R}_{2} \otimes \boldsymbol{\Lambda}_{1}+\mathbf{R}_{1} \otimes \boldsymbol{\Lambda}_{2}-k^{2} \mathbf{R}_{1} \otimes \boldsymbol{\Lambda}_{1}\right. \\
\left.-\mathbf{e}_{n_{r}}^{n_{r}} \mathbf{e}_{n_{r}}^{n_{r} T} \otimes \boldsymbol{\Lambda}_{M}\right)\left(\mathbf{I}_{n_{r}} \otimes \mathbf{Q}\right) \mathbf{u}=\mathbf{f},
\end{gathered}
$$

where $\boldsymbol{\Lambda}_{i}, i=1,2$ and $\boldsymbol{\Lambda}_{M}$ are diagonal matrices consisting of eigenvalues of the corresponding circulant matrices and $\mathbf{Q}$ is the Fourier matrix with 
$i j$ th component given by $e^{2 \pi i i j / n_{\theta}} \cdot \mathbf{Q}^{H}$ is the Hermitian conjugate of $\mathbf{Q}$. The column vectors of $\mathbf{Q}$ are the eigenvectors of the circulant matrices and $\mathbf{I}_{n_{r}}$ is the identity matrix of size $n_{r}$. Then, the system reduces to $n_{\theta}$ independent tri-diagonal systems

$$
\begin{gathered}
\left(\lambda_{1}^{j} \mathbf{R}_{2}+\lambda_{2}^{j} \mathbf{R}_{1}-k^{2} \lambda_{1}^{j} \mathbf{R}_{1}-\lambda_{M}^{j} \mathbf{e}_{n_{r}}^{n_{r}} \mathbf{e}_{n_{r}}^{n_{r} T}\right) \hat{\mathbf{u}}^{j}=\hat{\mathbf{f}}^{j}, \\
j=0,1, \cdots, n_{\theta}-1
\end{gathered}
$$

where $\hat{\mathbf{u}}^{j}$ and $\hat{\mathbf{f}}^{j}$ are the vectors composed of the $j$ th components from the $n_{r}$ blocks of the discrete Fourier transform of $\mathbf{u}$ and $\mathbf{f}$ respectively. The discrete Fourier transforms can be performed efficiently by using FFT and the solution vector $\mathbf{u}$ is obtained by inverse FFT. Here, one needs only the knowledge of the eigenvalues of the matrices $\mathbf{T}_{\mathbf{i}}, i=1,2$ and $\mathbf{M}$. These are circulant matrices for which simple analytical expressions of the eigenvalues can be obtained. For example, for a circulant matrix of the form $\left[c_{0}, c_{1}, \cdots, c_{n-1}\right]$, the $j$ th eigenvalue is given by $\lambda^{j}=\sum_{k=0}^{n-1} c_{k} e^{-2 \pi \mathbf{i} j k / n}$.

For a general non-circular obstacle, we use the fictitious domain method to solve the original problem where the matrix $B$ in this subsection is used as a preconditioner (see for details Heikkola et al. [1998] and Nasir [2003]).

\subsection{A Mixed-Type Method}

As we will see later, to apply the standard finite element method to the exact DtN mapping, we need to compute an infinite sum which should be truncated in some way. In this section, we introduce an alternative way to approximate the DtN mapping by the idea of mixed method.

Let $a(.,$.$) be a bounded V$-elliptic sesqui-linear form defined in a Hilbert space $V$ with inner-product $(.,)_{V}$ and let $A$ be a corresponding operator from $V$ into $V$ given as $a(u, v)=(A u, v)_{V}$. Let $b(u, v)$ be another bounded sesquilinear form in $V$ and let $B$ be defines as $b(u, v)=(B u, v)_{V}$. Assume that $B$ is one-to-one and positive. Let $L$ be $L=B^{-1} A$ with $\mathcal{D}(L)=\{u \mid A u \in R(B)\}$. Consider the problem to find $u \in V$ for a given $f \in V$ such that

$$
L^{n} u=f,
$$

where $n$ is a non-negative integer. By introducing intermediate unknowns $p_{j}, j=1,2, \cdots, n-1$, the problem (12) can be equivalently written as

$$
A p_{n-1}=B f, A p_{n-2}-B p_{n-1}=0, \cdots, A p_{1}-B p_{2}=0, A u-B p_{1}=0 .
$$

The weak formulation of this problem is to find $p_{j} \in V, j=0,1, \cdots, n-1$ such that for each $j=0,1, \cdots, n-1$,

$$
a\left(p_{j}, q_{j}\right)-b\left(p_{j+1}, q_{j}\right)=0, \quad \forall q_{j} \in V,
$$

where $u=p_{0} \in V$ and $p_{n}=f \in V$. When we pose the problem in a finite dimensional subspace $V_{h}$ of $V$ and use a given basis $\left\{\psi_{i}\right\}_{0}^{N_{h}}$, the approximate 
problem is given by the block matrix equation in $C^{N_{h}}, N_{h}=\operatorname{dim} V_{h}$ of the form

$$
[A]_{h} P_{j}-[B]_{h} P_{j+1}=0, \quad j=0,1, \cdots, n-1,
$$

where the matrices $[A]_{h}$ and $[B]_{h}$ are given by

$$
\left([A]_{h}\right)_{m n}=a\left(\psi_{n}, \psi_{m}\right), \quad\left([B]_{h}\right)_{m n}=b\left(\psi_{n}, \psi_{m}\right)
$$

and $P_{j}=\left[P_{j 1}, \cdots, P_{j N_{h}}\right]^{T}$ are the vector of coefficients in the representation of $p_{j, h} \in V_{h}$ with respect to the basis: $p_{j, h}=\sum_{k=0}^{N_{h}} P_{j k} \psi_{k}$.

Eliminating the block components $P_{j}, j=1,2, \ldots n-1$, we have

$$
[A]_{h}\left([B]_{h}^{-1}[A]_{h}\right)^{n-1} U=\left([A]_{h}[B]_{h}^{-1}\right)^{n}[B]_{h} U=F
$$

where $U=P_{0}$ and $F=P_{n}$. The mixed-type approximation matrix of $L^{n}$ is then given by $\left([A]_{h}[B]_{h}^{-1}\right)^{n}[B]_{h}$.

From these observations, we introduce a mixed-type approximation for a function $M(L)$ of operator $L$ as follows.

Definition (A Mixed-Type Method) Let $V_{h}$ be a finite dimensional subspace of $V$. Then, a mixed-type approximation matrix for the sesqui-linear form $\langle u, v\rangle_{M}=b(M(L) u, v)$, denoted by $[M(L)]_{h}$, with respect to a given basis of $V_{h}$ is defined as

$$
[M(L)]_{h}=M\left([A]_{h}[B]_{h}^{-1}\right)[B]_{h} .
$$

\subsection{Application to Radiation Boundary Condition}

Let $S$ be the unit circle. We choose the space $V=H^{1}(S)$ with the innerproduct $(u, v)_{V} \equiv\left(u^{\prime}, v^{\prime}\right)+(u, v)$ where $(.,$.$) is the L_{2}$-inner-product: $(u, v) \equiv$ $\int_{0}^{2 \pi} u \bar{v} d \theta$. Let us define sesqui-linear forms as

$$
a(u, v) \equiv\left(u^{\prime}, v^{\prime}\right) \quad \text { and } \quad b(u, v) \equiv(u, v) .
$$

Then the operator $L$ becomes $D^{2}$.

We denote by $\operatorname{Circ}(a, b, c)$ a circulant matrix for which the main diagonal is formed by $b$ and lower and upper diagonals are formed by $a$ and $c$ respectively. For the mixed-type approximation matrix given by piecewise linear continuous Lagrangian basis functions defined on a uniform partition with interval size $h_{\theta}=2 \pi / n_{\theta}$, we have

$$
[A]_{h}=\frac{1}{h_{\theta}} \operatorname{Circ}(-1,2,-1) \quad \text { and } \quad[B]_{h}=\frac{h_{\theta}}{6} \operatorname{Circ}(1,4,1) .
$$

The eigenvalues of $[B]_{h}$ and $[A]_{h}$ are then given by

$$
\lambda_{B}^{j}=\frac{h_{\theta}}{3}\left(2+\cos j h_{\theta}\right) \text { and } \lambda_{A}^{j}=\frac{2}{h_{\theta}}\left(1-\cos j h_{\theta}\right) .
$$


The eigenvalues of $[M(L)]_{h}=\left[M\left(D^{2}\right)\right]_{h}$ is hence given as

$$
\lambda_{M}^{j}=M\left(\nu_{j}^{2}\right) \lambda_{B}^{j} \quad \text { with } \quad \nu_{j}^{2}=\frac{\lambda_{A}^{j}}{\lambda_{B}^{j}}=\frac{1}{h_{\theta}^{2}} \frac{6\left(1-\cos j h_{\theta}\right)}{2+\cos j h_{\theta}} .
$$

For the exact DtN mapping (8) and the corresponding bilinear form $\langle u, v\rangle_{M}$, the eigenvalues of the mixed-type approximation matrix is

$$
\lambda_{D t N}^{j}=k R \frac{H^{\prime}\left(k R ; \nu_{j}\right)}{H\left(k R ; \nu_{j}\right)} \lambda_{B}^{j} .
$$

This can be directly computed without the Hankel function and its derivative by using the continued fraction( see Nasir [2003])

$$
x \frac{H^{(1)^{\prime}}(x ; \nu)}{H^{(1)}(x ; \nu)}=\mathbf{i} x-\frac{1}{2}+\mathbf{i} \frac{(1 / 2)^{2}-\nu^{2}}{2(x+\mathbf{i})+} \frac{(3 / 2)^{2}-\nu^{2}}{2(x+2 \mathbf{i})+} \ldots
$$

where $x=k R$.

We can prove the theoretical convergence and the practical computational efficiency of this type of mixed method in the case of the DtN mapping (see Nasir et al. [2003]).

Here we remark the standard finite element method for the DtN mapping (see for details Nasir [2003]). When we use piecewise linear continuous functions $\left\{\phi_{i}\right\}_{0}^{n_{\theta}-1}$ as the basis for the finite element approximation, the $i j$ th entry in the finite element matrix is given by

$$
\begin{aligned}
& \left\langle\phi_{j}, \phi_{i}\right\rangle_{M}=\frac{1}{2 \pi} \sum_{n=-\infty}^{\infty} R M\left(n^{2}\right)\left(\phi_{j}, e_{n}\right) \overline{\left(\phi_{i}, e_{n}\right)} \\
= & \frac{R M(0) h_{\theta}^{2}}{2 \pi}+\frac{1}{\pi} \sum_{n=1}^{\infty} R M\left(n^{2}\right) \frac{4\left(1-\cos n h_{\theta}\right)^{2}}{n^{4} h_{\theta}^{2}} \cos n h_{\theta}(j-i) .
\end{aligned}
$$

One advantage of the mixed method is that there is no infinite sum in the method and hence there is no ambiguity how to truncate the infinite series which exists in the standard finite element method. Furthermore, the computational cost of the mixed method is smaller than the one for the standard finite element method, although the performance is similar to the standard one which is known to be the best among all the methods using artificial boundary conditions.

\section{Numerical Simulation of Voice Generation}

The basic mathematical models of voice generation are the standard wave equation in unbounded domain and the corresponding Helmholtz equation for the frequency response problem. 
As for the boundary conditions of the problem, we impose the inhomogeneous Dirichlet or Neumann boundary condition on the vocal cord part as the sound source and the homogeneous Neumann condition on the rest part of the vocal tract surface which can be modeled as the rigid wall. Imposing the artificial Dirichlet to Neumann type boundary condition on the common artificial boundary between the interior and exterior regions, we can reduce the problem into the one inside the artificial boundary.

In the voice generation process of vowels, vocal cord vibrates nonlinearly and makes a (linear) sound wave with a fixed pitch (or a basic time frequency) having a characteristic Fourier spectrum. Articulation organs like tongue and jaw make a modification of the Fourier spectrum as a kind filter and produce different kind of vowels depending on different shapes of oral cavities (see Figure 1 for the two-dimensional model shape of Japanese and also Russian vowel $/ i /)$.

Formants are the peaks of the frequency response function. The cognition of different vowels are made by detecting the position of formants according to the experiments. Especially the lowest two or three formants are very important for the cognition.

For the general background of the voice generation problem in human speech, see Flanagan [1972] and Furui [1989].

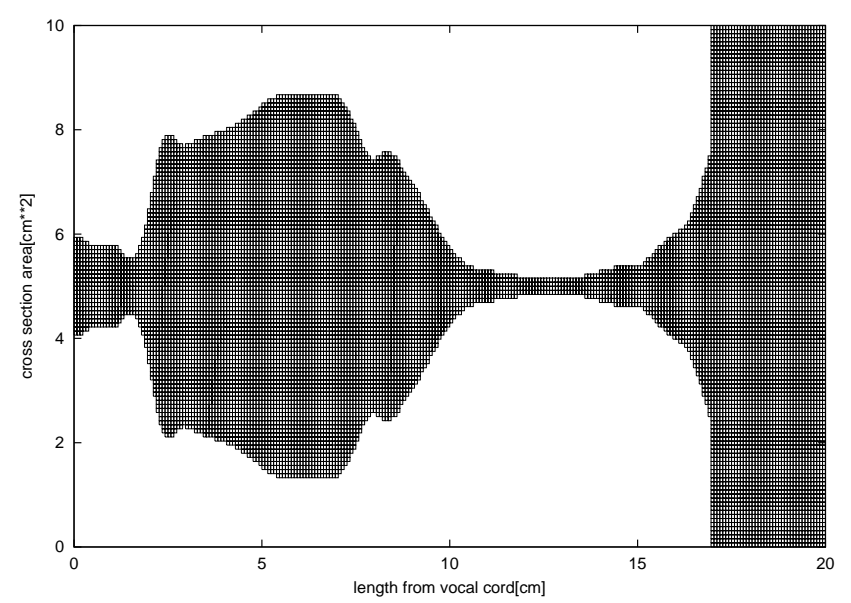

Fig. 1. Two-dimensional shape of vocal tract for vowel /i/

\subsection{Mathematical Model of Voice Generation}

Let $\Omega$ be a domain in $R^{n}$ consisting of the bounded part $\Omega_{i}$ which corresponds to the vocal tract and the exterior unbounded part $\Omega_{e}$ : 


$$
\bar{\Omega}=\overline{\Omega_{i}} \cup \overline{\Omega_{e}} .
$$

The sound pressure $u(t, x)$ in $\Omega$ with the source terms $f, g$ satisfies the following wave equation:

$$
\begin{aligned}
\left(\frac{\partial^{2}}{\partial t^{2}}-c^{2} \Delta\right) u(t, x) & =f(t, x) \quad \text { in } R \times \Omega, \quad \Delta=\sum_{i=1}^{2} \frac{\partial^{2}}{\partial x_{i}^{2}}, \\
\frac{\partial u}{\partial n}(t, x) & =g(t, x) \quad \text { on } R \times \partial \Omega,
\end{aligned}
$$

with sound velocity $c$, and $\partial / \partial n$ denotes the outward unit normal on the boundary $\partial \Omega$ of $\Omega$.

The time harmonic problem associated with this equation with the time frequency $\omega$ is formulated as the reduced wave equation or the Helmholtz equation for the stationary pressure $u(x)$ :

$$
\begin{aligned}
\left(-\Delta-k^{2}\right) u(x) & =f(x) \text { in } \Omega, \quad k=\omega / c \\
\frac{\partial u}{\partial n}(x) & =g(x) \text { on } \partial \Omega .
\end{aligned}
$$

We have to impose an appropriate radiation condition at infinity that exclude unphysical incoming waves. In the following, we treat the problem in one or two dimensional case.

\subsection{One and Two Dimensional Vocal Tract Models and DtN Mapping}

To reduce the problem in unbounded domain into the one in a bounded domain $\Omega_{i}$, we introduce an artificial boundary condition on the common boundary $\Gamma$ of the exterior and the interior domains: $\Gamma \equiv \overline{\Omega_{i}} \cap \overline{\Omega_{e}}$.

For this purpose, we firstly give the Dirichlet data $\left.u\right|_{\Gamma}$ on $\Gamma$ and solve the exterior problem, then we have the directional derivative $\partial u / \partial n$ of the solution along the exterior normal. This mapping form $\left.u\right|_{\Gamma}$ to $\partial u /\left.\partial n \equiv M u\right|_{\Gamma}$ is the Dirichlet to Neumann mapping in this case.

The original problem then becomes equivalent to boundary value problem in the interior domain:

$$
\begin{aligned}
\left(-\Delta-k^{2}\right) u & =0 \text { in } \Omega_{i} \\
\frac{\partial u}{\partial n} & =g \text { on } \partial \Omega \cap \overline{\Omega_{i}} \\
\frac{\partial u}{\partial n} & =\left.M u\right|_{\Gamma} \text { on } \Gamma .
\end{aligned}
$$

In the tubular exterior infinite domain case (see Kako and Kano [1999]), we have 


$$
\begin{aligned}
& \left.M u\right|_{\Gamma}=\sum_{n=0}^{\infty} \zeta_{n} C_{n}\left(\left.u\right|_{\Gamma}\right) c_{n}(y) \\
& C_{n}\left(\left.u\right|_{\Gamma}\right)=\int_{0}^{y_{0}} u(L, y) c_{n}(y) d y \quad(n \geq 0), \\
& c_{n}(y)= \begin{cases}\sqrt{\frac{1}{y_{0}}} & (n=0) \\
\sqrt{\frac{2}{y_{0}}} \cos \left(\frac{n \pi}{y_{0}} y\right) & (n \geq 1),\end{cases} \\
& \zeta_{n}=\left\{\begin{array}{lll}
i \xi_{n}, & \xi_{n}=\left\{k^{2}-\left(\frac{n \pi}{y_{0}}\right)^{2}\right\}^{1 / 2}, & 0 \leq n<\frac{y_{0}}{\pi} k \\
-\eta_{n}, & \eta_{n}=\left\{\left(\frac{n \pi}{y_{0}}\right)^{2}-k^{2}\right\}^{1 / 2}, & \frac{y_{0}}{\pi} k \leq n .
\end{array}\right.
\end{aligned}
$$

On the other hand, the following one dimensional simpler mathematical model is also used for the voice simulation problem and it is called the Webster's horn equation which is derived from the conservation law:

$$
-\frac{\partial v}{\partial t}=\frac{A(x)}{\rho} \frac{\partial u}{\partial x}, \quad-\frac{\partial u}{\partial t}=\frac{\rho c^{2}}{A(x)} \frac{\partial v}{\partial x}
$$

as

$$
\frac{\partial^{2} u}{\partial t^{2}}-\frac{1}{A(x)} c^{2} \frac{\partial}{\partial x}\left(A(x) \frac{\partial u}{\partial x}\right)=0
$$

here $A(x)$ denotes the cross sectional area of the vocal tract. The corresponding time harmonic problem for each wave number $k=\omega / c$ is given as

$$
\begin{gathered}
-\frac{1}{A(x)} \frac{d}{d x}\left(A(x) \frac{d u}{d x}\right)-k^{2} u=0, \\
\frac{d u}{d x}(0)=1, \quad \frac{d u}{d x}(L)=i k u(L) .
\end{gathered}
$$

The finite element method can be applied in the similar way as in the case of obstacle problem presented in Section 1, and we can obtain some theoretical results for the convergence of the numerical solution to the exact one in case of the standard method (see Kako and Kano [1999] ). We can also use the mixed type method in this case.

\subsection{Complex Resonance Eigenvalue and Variational Formula}

For the numerical simulation of voice generation, we change the frequency $\omega$ and hence the wave number $k$, and calculate the amplification factor computed as the sound pressure at some observation point for the given unit volume velocity of sound at the vocal cord position where the sound originates. The observation point should be located outside of the vocal tract region, and, in the following examples, we choose the point just on the artificial boundary.

This correspondence between the frequency and the amplification factor is the frequency response function or the formant curve. The peaks of this 
curve are called formants. A typical example of the frequency response function is shown in Figure 2 in the case of vowel / $i$ / for one and two dimensional cases. In two-dimensional computation based on DtN mapping, we used the shape in Figure 1, and applied the finite element method with $392 \times 196$ background rectangular mesh and corresponding triangulation. We used the usual piecewise linear continuous basis functions for discretization. One-dimensional computation is performed similarly for the area function $A(x)$ corresponding to Figure 1. We plotted the results for the frequency range from 0 to $4.7 \mathrm{KHz}$ and the pressure $u$ is shown in the logarithmic scale.

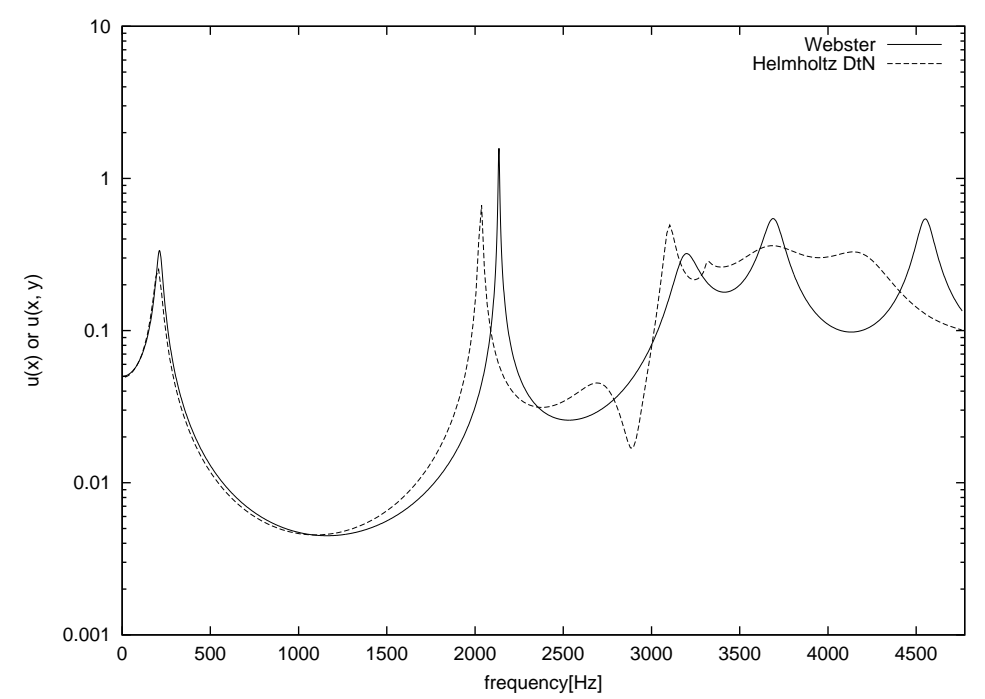

Fig. 2. Frequency response function for vowel /i/

If we change the shape of the vocal tract or the cross section area, the frequency response varies (see Kent and Read [1992] for the heuristic perturbation theory for vocal tract shape and formant curve). The frequency response curve is determined through the complex eigenvalues and its eigenfunction of the Helmholtz equation with the complex frequency $\omega$ extended into the lower half complex plane.

We show one-dimensional numerical example for this correspondence between the complex poles and the frequency response function in Figure 3 where we moved the shape of vocal tract from the neutral shape with constant cross sectional area to the one of $/ i /$ as is illustrated in Figure 4. These complex poles are calculated by the iteration process of line search in real and imaginary directions alternatively. We took the starting values of the iteration to be the eigenvalues for the previous shape.

As shown in Figure 4, the formants are closely related to the resonant complex eigenvalues. Especially, if the real part of a resonance complex eigenvalue 
moves, the position of the formant moves correspondingly to the same direction. Furthermore, the imaginary part of the complex eigenvalue corresponds to the strength of the formant. Hence, to control the positions of formants, it would be an efficient way to vary the positions of the resonant complex eigenvalues. As for the mathematical treatments of resonance poles and related topics, refer the works by Lenoir et al. [1992] and Poisson [1995].

In this respect, the variational formula for the resonant complex eigenvalues will be useful to design the format curve to simulate the natural formant curve of human beings. Under the assumption that the eigenvalues and the eigenfunctions move smoothly with respect to the variation of shape of the vocal tract, we can obtain the variational formula as follows.

In one-dimensional case, let $k$ and $u(x ; k)$ satisfy the one-dimensional homogeneous Webster's horn equation which is the complex eigenvalue problem for $k$ and $u$ :

$$
\begin{gathered}
-\frac{d}{d x}\left(A(x) \frac{d u}{d x}\right)-k^{2} A(x) u(x)=0, \\
\frac{d u}{d x}(0)=0, \quad \frac{d u}{d x}(L)=i k u(L) .
\end{gathered}
$$

Then the variational formula is given as follows with respect to the variation $(\delta A)(x)$ of the cross section area $A(x)$ of the vocal tract:

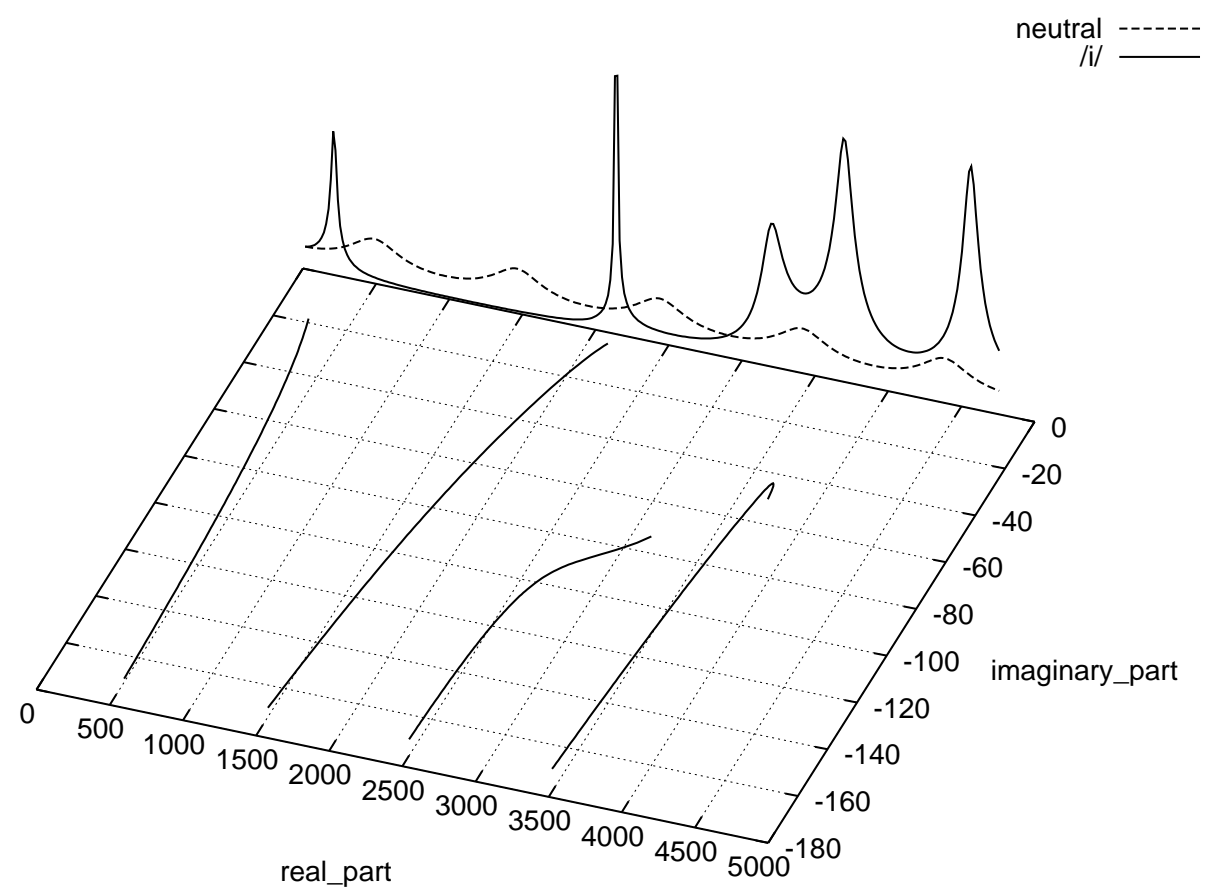

Fig. 3. Correspondence between complex eigenvalues and frequency response 


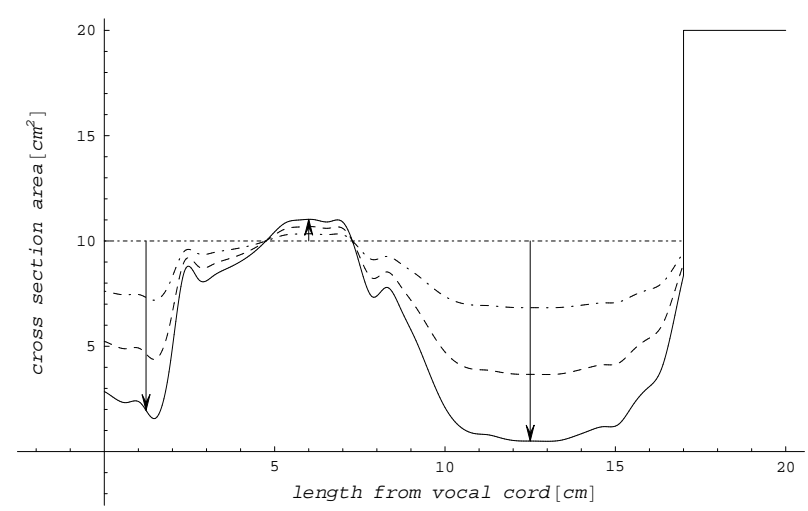

Fig. 4. Changing of area from the neutral shape to /i/

$$
\delta k=\frac{\int_{0}^{L}(\delta A)(x)\left\{\left(\frac{d u}{d x}(x)\right)^{2}-k^{2} u(x)^{2}\right\} \mathrm{d} x-i k(\delta A)(L) u(L)^{2}}{2 k \int_{0}^{L} A(x) u(x)^{2} \mathrm{~d} x+i A(L) u(L)^{2}} .
$$

We can prove this formula multiplying the equation by the solution and doing the integration by parts.

We show in Figure 5 a numerical example which corresponds to the third formants and the variational formula for the corresponding complex eigenvalue. There is a good coincidence between the tangent directions and the values computed by the variational formula which are illustrated by the vectors with arrow on the eigenvalue trajectory.

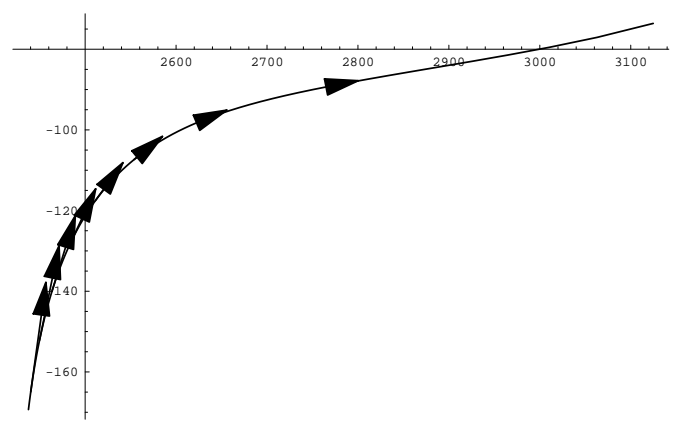

Fig. 5. Complex vectors by variational formula on eigenvalue trajectory 
For two dimensional problem, let $n$ be the outward normal and $\psi(x)$ be a function on the boundary, and move the boundary to the direction of the normal as $\epsilon \psi n$.

Firstly we consider the case of completely elastic boundary: $u=0$ on $\partial \Omega$. The variational derivative of the resonant complex eigenvalue is then given as

$$
\frac{\delta k}{\delta \psi}=\frac{-\int_{\partial \Omega}\left(\frac{\partial u}{\partial n}\right)^{2} \psi \mathrm{d} \sigma}{2 k \int_{\Omega} u(x)^{2} \mathrm{~d} x+\int_{\Gamma}\left(\left(\frac{\delta M}{\delta k}\right) u\right) u \mathrm{~d} \theta}
$$

Next we consider the case of rigid boundary: $\partial u / \partial n=$ on $\partial \Omega$. In this case the variational formula is given as:

$$
\begin{gathered}
\frac{\delta k}{\delta \psi}=\frac{\int_{\partial \Omega}\left\{n_{1}^{2} \frac{\partial^{2} u}{\partial x_{1}^{2}}+2 n_{1} n_{2} \frac{\partial^{2} u}{\partial x_{1} \partial x_{2}}+n_{2}^{2} \frac{\partial^{2} u}{\partial x_{2}^{2}}\right\} \psi u \mathrm{~d} \sigma}{2 k \int_{\Omega} u(x)^{2} \mathrm{~d} x+\int_{\Gamma}((\delta M / \delta k) u) u \mathrm{~d} \theta} \\
=\frac{\int_{\partial \Omega}\left\{\left(\frac{\partial u}{\partial \sigma}\right)^{2}-k^{2} u^{2}\right\} \psi u \mathrm{~d} \sigma+\int_{\partial \Omega} \frac{\partial \phi}{\partial \sigma} u \frac{\partial u}{\partial \sigma} \mathrm{d} \sigma}{2 k \int_{\Omega} u(x)^{2} \mathrm{~d} x+\int_{\Gamma}((\delta M / \delta k) u) u \mathrm{~d} \theta}
\end{gathered}
$$

Now, we modify the Dirichlet to Neumann mapping on the radiation boundary rather artificially multiplying the homotopy parameter $\alpha \in[0,1]$ : $\frac{\partial u}{\partial n}=\alpha M u$. Then, if $\alpha=0$, the boundary condition becomes the homogeneous Neumann condition, and hence the eigenvalues are all real and discrete. If we move $\alpha$ from 0 to 1 , we may establish the relationship between real eigenvalues and the resonant complex eigenvalues. In the case of $\alpha=0$, the variation becomes pure imaginary, and resonance pole moves into the lower complex plane vertically to the real line.

\section{Concluding Remarks}

We formulated the stationary wave propagation phenomena in unbounded region outside an obstacle via the Helmholtz equation with appropriate boundary conditions. We introduced two kinds of finite element approximation based on the standard method and a mixed type one. The later has better numerical performance in the sense that it needs less computational cost and smaller memory requirement. From the theoretical error analysis, we can conclude the convergence of the numerical solution to the exact one.

We computed one dimensional as well as two dimensional voice generation problem which give reasonable frequency response curves. We then introduced variational formulas for the complex eigenvalues or the resonance poles for two 
dimensional as well as one dimensional problems. We suggested a procedure to design the frequency response curves based on the variational formula and presented a numerical example to show the validity of our method.

\section{References}

A. Bayliss and E. Turkel. Radiation boundary conditions for wave-like equations. Comm. Pure and Appl. Math., 33(6):707-725, 1980.

B. Engquist and A. Majda. Absorbing boundary conditions for the numerical simulation of waves. Math. Comp., 31(139):629-651, 1977.

B. Engquist and A. Majda. Radiation boundary conditions for acoustic and elastic wave calculations. Comm. Pure Appl. Math., 32(3):313-357, 1979.

O. G. Ernst. A finite-element capacitance matrix method for exterior helmholtz problems. Numer. Math., 75(2):175-204, 1996.

K. Feng. Finite element method and natural boundary reduction. In Proceeding of the International Congress of Mathematicians, Warsaw, Poland, 1983.

J. Flanagan. Speech analysis, synthesis, and perception. Springer, Berlin-New York, 1972.

S. Furui. Digital speech processing, synthesis, and recognition. Marcel Dekker, 1989.

E. Heikkola, Y. A. Kuznetsov, P. Neittaanmaki, and J. Toivanen. Fictitious domain methods for the numerical solution of two-dimesional scattering problems. J. Comput. Phys., 145:89-109, 1998.

T. Kako and T. Kano. Finite element method for the helmholtz equation and numerical simulation of the wave propagation in vocal tract. In GAKUTO Int. Series Math. Sci. and Appli., volume 12, pages 55-63, 1999.

R. Kent and C. Read. The acoustic analysis of speech. Singular Publ. Group, San Diego, 1992.

M. Lenoir, M. Vullierme-Ledard, and C. Hazard. Variational formulations for the determination of resonant states in scattering problems. SIAM J. Math. Anal., 23(3):579-608, 1992.

H. Nasir. A mixed type finite element method for radiation and scattering problems with applications to structural-acoustic coupling problem in unbounded region. PhD thesis, The University of Electro-Communications, Japan, 2003.

H. Nasir, T. Kako, and D. Koyama. A mixed-type finite element approximation for radiation problems using fictitious domain method. J. Comput. Appl. Math., 52:377-392, 2003.

O. Poisson. Étude numérique des pôles de résonance associés à la diffraction d'ondes acoustiques et élastiques par un obstacle en dimension 2. $M^{2} A N$, 29(2):819-855, 1995. 